\title{
A Simulation Study on The Simulated Annealing Algorithm in Estimating The Parameters of Generalized Gamma Distribution
}

\author{
Amani Idris A. Sayed ${ }^{1,2 *}$ and Sabri Shamsul Rijal Muhammad ${ }^{1}$ \\ ${ }^{1}$ School of Mathematical Sciences, University Sains Malaysia, Pulau Pinang, 11800, Malaysia \\ ${ }^{2}$ Department of Mathematics, Jazan University, Jazan, 45142, Saudi Arabia \\ *Corresponding Author e-mail: amani.sayed1983ayahoo.com
}

\begin{abstract}
This study proposed a hybrid computational model by incorporating Simulated Annealing algorithm (SA) in the maximum likelihood in estimation the parameters of Generalized Gamma Distribution (GGD). The purpose is to improve the searching capacity of maximum likelihood estimator for Generalized Gamma Distribution (GGD). A simulated Annealing algorithm (SA) is one of the global search heuristics computational approach inspired by the metallurgical process, in which metal is rapidly heated to a high temperature, then cooled slowly until it reaches the lowest-energy state, increasing its strength and making it easier to work with. It has been applied in approximating global optimization in a large search space for various optimization problems. This study employs a Simulated Annealing algorithm (SA) to improve the global search capacity of maximum likelihood methods (MLE) in estimating the parameters of the Generalized Gamma Distribution (GGD). The Generalized Gamma Distribution (GGD) constitutes an extensive family that contains nearly all of the most commonly used distributions including the exponential, Weibull, and lognormal distribution. The performances of the proposed estimation method are computed based on their biases and mean square errors through a simulation study. The study reveals that the Simulated Annealing algorithm (SA) performs better than the classical estimators in estimating the parameters of Generalized Gamma Distribution (GGD).
\end{abstract}

Keywords

Simulated Annealing Algorithm, Generalized Gamma Distribution, Parameters Estimation Methods, Log-Likelihood Function.

Received: 28 October 2021, Accepted: 08 January 2022

https://doi.org/10.26554/sti.2022.7.1.84-90

\section{INTRODUCTION}

The Gamma Distribution (GD) is one of the most important statistical distributions for its widely used in many fields of data modelling and forecasting of various phenomena. It is useful in handling large scales of data due to its flexibility to be transformed to other distributions such as exponential and normal distributions. The important features of the Generalized Gamma Distribution (GGD) made it useful in various areas of research which include business, engineering and other lifetime analysis (Lawless, 2003; Tang and Cheong, 2004; Eric et al., 2020). The probability density function of the gamma distribution is given as.

$$
f_{X_{K}}(x, \alpha, \theta)= \begin{cases}\frac{1}{\Gamma(\alpha)}\left[\frac{1}{\theta}\right]^{\alpha} x^{\alpha-1} e^{\frac{x}{\theta}}, & x>0 \\ 0, & \text { otherwise }\end{cases}
$$

where $a$ and $q$ are the shape and scale parameters of the gamma distribution model respectively. We employ a Generalized Gamma Distribution (GGD) in this work, which was established by Amoroso (1925). The gamma distribution is among the most widely statistical distribution employed in modelling a different kind of lifetime data set with application in modelling different phenomena characterized with monotone failure rates. It requires a little measure of parameters for learning. The first generalization of the gamma distribution can be found in Amoroso (1925), which analyzed and applied a GGD to fitting the income rates based on the GGD with four parameters has been studied by Johnson et al. (1995). The study on the four gamma distribution was later reduced to the GGD according to Stacy (1962) by setting the location parameter to zero. Mudholkar and Srivastava (1993) used the exponentiated method to derive the gamma distribution model for data anlysis. Stacy (1962) developed the generalized version of the gamma distribution based three-parameters. Agarwal and Al-Saleh (2001) modelled the distribution of hazard rates using the gamma distribution model. Similar distribution was emplyed by Balakrishnan and Peng (2006) in creating a generalized version of gamma distribution to modelling frailty 
data. Another sort of GGD suggested by Nadarajah and Gupta (2007) was used to fit drought data. Another generalization of distribution by Stacys used an exponentiated approach to GGD model and used it to lifetime and survival analysis (Ortega et al., 2012). A study by Chen et al. (2017) employed a generalized version of the gamma distribution (GGD) with three parameters in modelling the flood frequency data analysis. Zhao et al. (2018) employed the three-parameter GGD model in calculating the statistical characteristics of high-resolution SAR pictures. Similarly, Mead et al. (2018) propose the modified version of GGD to evaluate greater flexibility and appropriateess in representing data from a practical standpoint, and they derived a variety of identities and properties of this distribution, which include explicit representations for lifetime expression.

Researchers in the fields of statistical distribution have tried a variety of methods in estimating the parameters of the GGD. This include, moment method (Khodabina and Ahmadabadib, 2010), quantile methods (Nagatsuka and Balakrishnan, 2012), modified moment methods (Dey et al., 2021), evolutionary algorithm (Yonar and Yapici, 2020), maximum likelihood (Hirose, 1995), Bayesian approach (Khodabina and Ahmadabadib, 2010; Pradhan and Kundu, 2011). However, because parameters involve leading to the same density function, it is difficult and challenging face by researchers in estimating the parameters of the generalized version of the gamma distribution using methods like moment methods and maximum likelihood. The likelihood equations must be solved simultaneously in the maximum likelihood technique. To explore other approach in estimating the parameter of the generalized version of the gamma distribution, conventional methods were developed. It also showcases the incapability of the conventional/traditional method such as moment method and maximum likelihood estimator to estimate the parameters of the proposed GGD distribution. However, the results are unappealing due to their intricacy of the traditional method. As a result, it appears that estimating the parameters of the GGD is quite challenging and not straightforward. There is the need to incorporating other search approach such as heuristics method to overcome such challenges and limitation associated with the conventional methods. In this study, a stochastic computational methods based on the Simulated Annealing algorithm (SA) has been incorporated to reduce the complexity involve in the searching for the optimal parameters of the generalized version of the gamma distribution model. In this study, a simulation study has been carried out according to the proposed model. The remainder of this paper is organized as follows: The simulated annealing algorithm and Generalize gamma distribution and its parameters have been presented in Section 2. A simulated annealing algorithm for optimization of gamma distribution parameters is studied in Section 3. The experimental results and with discussion have been presented in Section 4. Conclusions and recommendation the of the study in Section 5 .

\section{SIMULATED ANNEALING ALGORITHM}

The Simulated Annealing algorithm (SA) aims to generate new solutions using a random process through a series of probability distributions. This random operation does not necessarily improve the objective function, but even so, it may be accepted (Franzin and Stützle, 2019). The algorithm was originally used as an optimization process in Metallurgical engineering to achieve the minimum energy by gradually reducing the atomic motion that reduces the uniformity of lattice defects, thereby reducing the metal temperature (Du and Swamy, 2016). The Simulated Annealing algorithm (SA) cannot be affected by any restraint at any local minimum and has an indulgent acceptance of any changes that arise in the objective function, thus its efficiency makes it useful in many fields, including finance and the mathematical and statistical sciences (Crama and Schyns, 2003; Orús et al., 2019; Abubakar and Sabri, 2021b; Abubakar and Sabri, 2021 a).

The acceptance probability in the $\mathrm{SA}$ algorithm can be written as,

$$
P=e^{\frac{-(\Delta Z)}{H}}
$$

Where $\Delta Z$ denote the increase in the objective function and $H$ represent the controlling parameter. Some studies including (Abbasi et al., 2006) and (Bertsimas and Tsitsiklis, 1993), presented the most general form of the Simulated Annealing algorithm (SA) which can be written in the following steps: i. Start with initial solutions $X=X_{0}$.

ii. Generate a random chance of the solutions $X$.

iii. Evaluate the objective function at the randomly generated solutions.

iv. Setting an initial controlling parameter $H=H_{0}$ and an approach to reduce it as the process goes.

The general form of the SA algorithm, which has been explained above, works toward minimizing the control parameter $H$, in adjusting the procedure which can be implemented in other problems. The purpose is to explore the feasibility of Simulated Annealing algorithm (SA) in estimating the parameters of proposed model in relation to conventional method. And to find out how the estimated parameters will maximize the log-likelihood function. To maximize the log-likelihood function, the controlling parameter $H$ in the algorithm must be maximized too, so the objective function should be multiplied by (-1) according to (Sabri and Sarsour, 2019). The determination of the control parameter and generating initial and neighbouring solutions based on the SA procedure should be carefully observed. Also, the data sample should be big enough to obtain an accurate result. The performance of the Simulated Annealing algorithm (SA) has been assessed according to Mean Absolute Error (MAE) based on the simulated data set for different sample size. The effectiveness of Mean Absolute Error (MAE) has been demonstrated in several studies, including (Peng and Yao, 2003; Franses, 2016; Frías-Paredes et al., 2018; Sayed and Sabri, 2022). The following section presents 
the generalised gamma distribution along with its parameters and statistical properties.

\subsection{Generalize Gamma Distribution and its Parameters} The most common pdf form of the generalized version of the gamma distribution (GGD) can be written as,

$$
f(t ; \alpha, \beta, c)=\frac{\beta}{\Gamma(\alpha) c}\left(\frac{t}{c}\right)^{\alpha \beta-1} e^{-\left(\frac{t}{c}\right)^{\alpha}}
$$

Where $\alpha>0$ and $\beta>0$ are the shape parameters, $c>0$ is the scale parameter and $\Gamma(\alpha)$ is the gamma function that defines as,

$$
\Gamma(\alpha)=\int_{0}^{\infty} x^{\alpha-1} e^{-x} d x
$$

Because such a distribution is associated with convergent difficulties of the MLE, some reparameterization with the parameters was used to alleviate these difficulties as follow,

$$
\begin{aligned}
& \mu=\ln (c)+\frac{1}{\beta} \ln \left(\frac{1}{\lambda^{2}}\right) \\
& \sigma=\frac{1}{\beta \sqrt{\alpha}} \\
& \lambda=\frac{1}{\sqrt{\alpha}}
\end{aligned}
$$

where $-\infty<\mu<\infty, \sigma>0,0<\lambda$. Based on this reparameterization and the values of the parameters, the GGD can include special cases of some distributions such as The Gamma Distribution (GD), The Exponential Distribution (ED), Weibull Distribution (WD), and The Lognormal Distribution (LD) as shown in Figure 1.

In this case, a continuous random variable $X_{k}$ taking a non-negative real value is said to follow the gamma distribution for $K$-years period. $X_{k}$ distributed using two parameters Gamma distribution $\alpha$ and $\theta$ with mean $=\alpha \theta$ and variance $=\alpha \theta^{2}$. Then, $K=2, \ldots, 4$, we impose a new growth rate, $\gamma$ by letting $X_{k}=(1+\gamma)^{k-1} X_{1}$. Because the growth rate $\gamma$ is associated with the scale of the data, this results from the development of the Gamma distribution to become a generalized gamma distribution as follows,

$$
f_{X_{K}}\left(X, \alpha,(1+\gamma)^{K-1} \theta\right)= \begin{cases}\frac{1}{\Gamma(\alpha)}\left[\frac{1}{(1+\gamma)^{K-1} \theta}\right]^{\alpha} x^{\alpha-1} e^{\frac{x}{(1+\gamma)^{K-1}}}, & x>0 \\ 0, & \text { otherwise }\end{cases}
$$

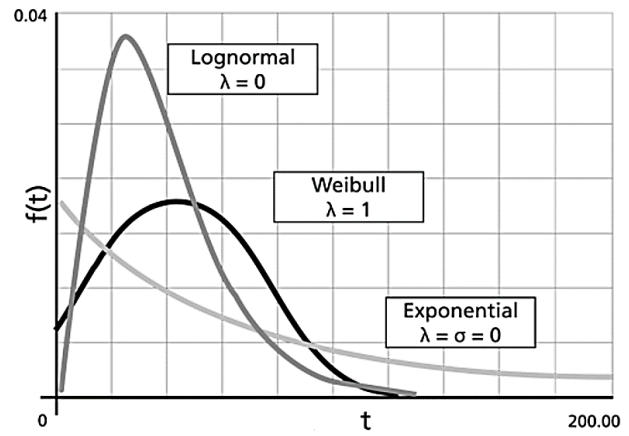

Figure 1. Generalized Gamma Distribution Based on The Different Parameters

With the parameters $\alpha$ and $(1+\gamma)^{K-1} \theta$ and having the mean $=\alpha(1+\gamma)^{K-1} \theta$ and variance $=\alpha\left[(1+\gamma)^{K-1} \theta\right]^{2}$. Estimating the parameters along with the growth parameter $\gamma$ of Equation (8) using the method of the moment is not an easy task because it's difficult to construct the values of them from the mean and variance equations unless we fix the value of one of them and solve for the other two.

From Equation (8), the likelihood function $(L)$ of $X_{K}$ is

$$
\begin{aligned}
L\left(\alpha,(1+\gamma)^{K_{1}} \theta \mid x_{K}\right)= & -\ln \left[\Gamma\left(\alpha_{K}\right)\right]-\alpha_{K} \ln \left((1+\gamma)^{K-1} \theta_{K}\right) \\
& +\ln \left(x_{K}^{\alpha_{K}-1}\right)-\frac{X_{K}}{(1+\gamma)^{K-1} \theta_{K}}
\end{aligned}
$$

and, the log-likelihood function is then be simplified as,

$$
\begin{aligned}
l\left(\alpha,(1+\gamma)^{K_{1}} \theta \mid x_{K}\right)= & -\sum_{K-1}^{K^{*}} \ln \left[\Gamma\left(\alpha_{K}\right)\right]-\sum_{K=1}^{K^{*}} \alpha_{K} \ln \left((1+\gamma)^{K-1} \theta_{K}\right) \\
& +\sum_{K=1}^{K^{*}} \ln \left(x_{K}^{\alpha_{K}-1}\right)-\sum_{K-1}^{K^{*}} \frac{X_{K}}{(1+\gamma)^{K-1} \theta_{K}}
\end{aligned}
$$

It is observed by various studies include (Abbasi et al., 2006) and Abubakar and Sabri (2021b) that the maximum likelihood approach used to estimate the three parameters of our generalized gamma model is a difficult process, this is because is hard to get a straightforward differentiation of the resulting $\log$-likelihood function in Equation (10). Therefore, it is mathematically challenging in searching for the optimal parameters of the generalized gamma distribution simultaneously from Equation (10) as differentiating the log-likelihood function towards $\alpha$ couldn't be derived directly. Therefore, it is easier to incorporate with another useful method such as the Simulated Annealing algorithm (SA) to search for the gamma distribution parameters, which works with the log-likelihood function as an objective function and aims to maximize it. The SA procedure 
use in estimating the parameters of the generalized gamma distribution has been presented in the following section.

\section{SIMULATED ANNEALING ALGORITHM FOR GAMMA PARAMETERS ESTIMATION}

In this work, a simulated annealing algorithm is used to estimate the parameters of the generalized gamma distribution and detect how close the estimated parameters are to the true ones. Furthermore, we observe how the estimated parameters are going to maximize the log-likelihood function. This procedure involves some steps, as it is shown in Figure 2.

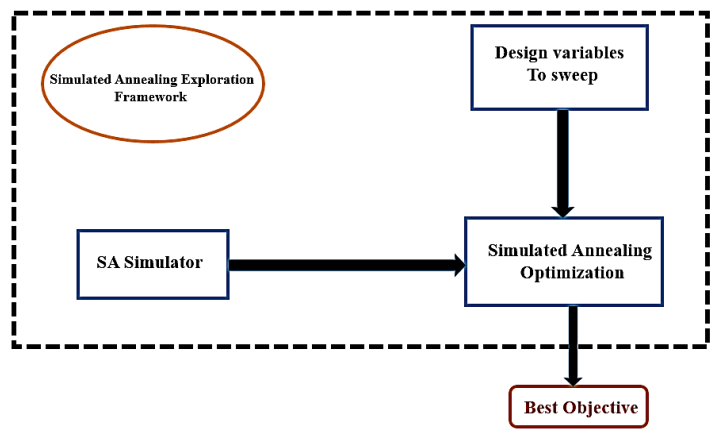

Figure 2. Block Diagram of The Proposed Framework

\section{Step 1: Variables Designing}

Start with generating the data sample randomly by simulation method using Microsoft Excel. From Equation (1) the cumulative density function of $X$ when $K=1$ is,

$$
F_{X_{1}}\left(X^{*}\right)=\int_{0}^{X^{*}} \frac{1}{\Gamma(\alpha)}\left(\frac{1}{\theta}\right)^{\alpha} x^{\alpha-1} e^{-\frac{x}{\theta}} d x=\operatorname{Gamma}\left(\alpha, \theta, x^{*}\right)
$$

If the value of $F_{x_{1}}\left(x^{*}\right)=p$, then we find $x$ as,

$$
\operatorname{Gamma}\left(\alpha, \theta, x^{*}\right)=p \rightarrow x^{*}=\operatorname{Gamma}^{-1}(\alpha, \theta, p)
$$

In Excel, the value of $p$ is computed as the function of $p=\operatorname{rand} a()$. The cumulative density function of $X$ when $K>1$ is,

$$
F_{X_{K}}\left(X^{*}\right)=\int_{0}^{X^{*}} \frac{1}{\Gamma(\alpha)}\left[\frac{1}{(1+\gamma)^{K-1} \theta}\right]^{\alpha} x^{\alpha-1} e^{-\frac{x}{(1+\gamma)^{K-1}}} d x
$$

and

$$
\operatorname{Gamma}\left(\alpha,(1+\gamma)^{K-1} \theta, x^{*}\right)=p
$$

Thus,

$$
x^{*}=\mathrm{Gamma}^{-1}\left(\alpha,(1+\gamma)^{K-1} \theta, p\right)
$$

So, in Microsoft Excel $x^{*}$ is generated and distributed using $\mathrm{CDF}$ of the gamma distribution to calculate the log-likelihood function in the following few steps:

1. Generate $p$ using the function $=\operatorname{rand}()$.

2. Select initial values for $\alpha, \theta, \gamma$.

3. Generate $x^{*}$ using the inverse gamma function $\mathrm{Gamma}^{-1}$ $\left(\alpha,(1+\gamma)^{K-1} \theta, p\right)$.

4. Find the cumulative gamma distribution $F_{X_{1}}\left(x^{*}\right)$ using the initial parameters.

5. Find the inverse $\log$ of $F_{X_{1}}\left(x^{*}\right)$ and calculate the loglikelihood function.

6. Estimate a set of 100 gamma parameters of $\alpha, \theta, \gamma$ using the simulated annealing method.

7. Find the cumulative gamma distribution $F_{X_{1}}\left(x^{*}\right)$ using the mean of the 100 estimated parameters.

8. Find the inverse $\log$ of $F_{X_{1}}\left(x^{*}\right)$ and calculate the $\log$ likelihood function.

\section{Step 2: Simulated Annealing Optimization}

As mentioned earlier, it is only helpful to incorporate a useful method such as simulated annealing to estimate Gamma parameters that maximize the log-likelihood function. The process of SA requires advanced programming, hence, in this study, MATLAB coding is used to estimate 100 values of the Gamma parameters. The SA algorithm is explained as follows:

1. Get a big enough sample of data $x^{*}$.

2. Determine the objective function $f=\left(l, x^{*}\right)$ where $l$ is the maximum likelihood.

3. Select controlling parameters for the $\mathrm{SA}$, for example, $H_{o}, H, A, B$ such that: while $H>H_{0}, H=A H$.

4. Select lower and upper bounds of random variables $l b=$ $[., .,],. u b=[., .,$.$] .$

5. Generate random values $a, b$, and $c$ within the lower and upper bounds.

6. Compute the likelihood function $l$ at random values.

7. Generate neighbour values $a_{1}, b_{1}$, and $c_{1}$ within the lower and upper bounds.

8. Compute the likelihood function $l_{1}$ at the neighbour values.

9. If $l_{1}>l$, then $l_{1}=l$, and $a=a_{1}, b=b_{1}, c=c_{1}$.

10. Else generate a random value $r \in(0,1)$.

11. If $e^{-\frac{\left(l_{1}-l\right)}{H}}>r$ then $a=a_{1}, b=b_{1}, c=c_{1}$.

12. Print 100 sets of $a, b$, and $c$ which are $\widehat{\alpha}, \widehat{\theta}$, and $\widehat{\gamma}$ respectively.

\section{EXPERIMENT RESULT AND DISCUSSION}

In this study, 4 sets with different samples sizes of 50,100 , 200, and 500 were generated using Microsoft Excel for the values of $K=2,3,4,5$ accordingly. The CDF of the gamma distribution with three initial parameters $(\alpha=4, \theta=0.3, \gamma=-0.05)$ was distributed across all data sets generated. Then the SA algorithm was used to further estimate 100 sets of the gamma parameters for each data sample size. The performance of the 
SA algorithm was evaluated using the Mean Absolute Error (MAE) of the estimated parameters $\widehat{\alpha}, \widehat{\theta}$, and $\widehat{\gamma}$ for each sample size as follows,

$$
\frac{1}{3}(|\widehat{\alpha}-\alpha|+|\widehat{\theta}-\theta|+|\widehat{\gamma}-\gamma|)
$$

The CDF of gamma distribution was calculated for each sample size with the initial parameters and the mean of the estimated parameters to see how close the distributions will be for the different data sets. Finally, the inverse of the maximum likelihood is calculated based on the initial parameters, then the average of the estimated parameters for each sample size to observe on how the means of our estimated parameters sets will maximize the maximum likelihood.

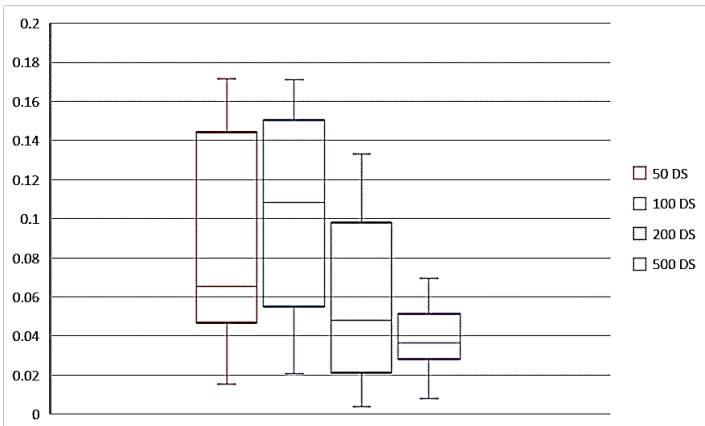

Figure 3. Box Plots of The MAE for Each Sample Size Sample $50,100,200$ and 500

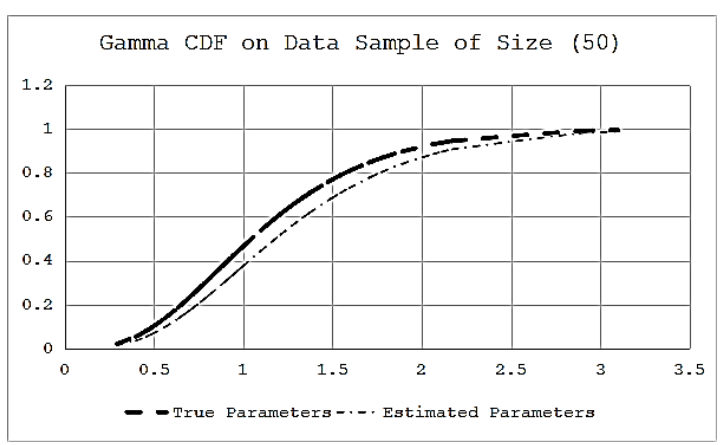

Figure 4. GDF of The GGD for 50 Sample Sizes

Table 1. Estimated Parameters of The GGD Via SA

\begin{tabular}{cccc}
\hline Sample size (n) & Mean of the estimate & $\begin{array}{l}\text { Likelihood at the } \\
\text { initial estimate }\end{array}$ & $\begin{array}{l}\text { Likelihood at the } \\
\text { Mean }\end{array}$ \\
\hline 500 & $4,0.31,-0.052$ & 504.7 & 524.27 \\
200 & $4.2,0.29,-0.051$ & 194.03 & 202.09 \\
100 & $4.2,0.27,-0.052$ & 93.25 & 125.57 \\
50 & $4.1,0.33,-0.051$ & 45.65 & 55.79 \\
\hline
\end{tabular}

According to box plots in Figure 3, the larger the sample size, the closer the average absolute error of the estimated parameters to zero. The sample of size 50 has a larger spread

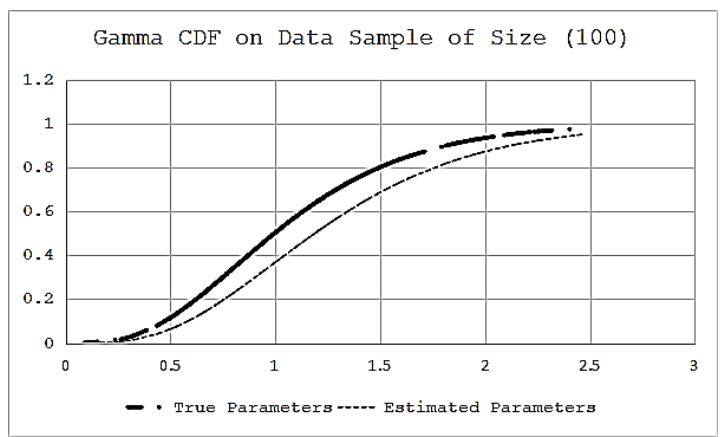

Figure 5. CDF of The GGD for 100 Sample Sizes

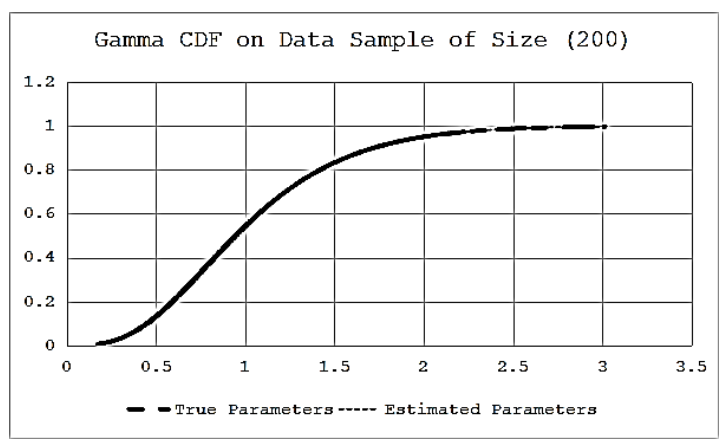

Figure 6. CDF of The GGD for 200 Sample Sizes

while the spread of the data gets smaller when the size of the data sample gets larger, which means that the 100 sets of estimated parameters $\widehat{\alpha}, \widehat{\theta}$, and $\widehat{\gamma}$ fall closer to the initial parameters the larger the data sample gets, which stresses the efficiency of the algorithm. Figures 4 to 7 , displayed the CDF of the GGD function based on the data set generated using both the initial parameters and the mean of the estimated parameters for each sample size. It can be observed that the estimated parameters of the generalized gamma distribution are getting closer to the true parameters as the sample size increases. Table 1 reports the mean of the estimate, the likelihood value of the mean and the initial estimate. It is seen that the Gamma accuracy of the estimated parameters is getting closer to the true parameters when the sample size becomes large. Moreover, the

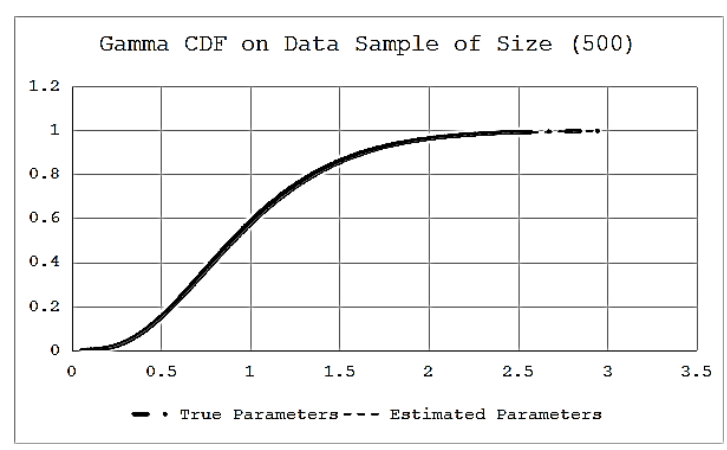

Figure 7. CDF of The GGD for 500 Sample Sizes 
mean estimated parameters using the SA algorithm maximize the likelihood function optimally. When it comes to parameter estimation for a Generalized Gamma Distribution (GGD), the classical method produces a lot of inaccuracies. Although the amount of inaccuracies in SA is low, the offered settings are occasionally surprising and unrealistic. This reveals that the simulated annealing algorithm can work optimally with maximum likelihood in estimating the parameters of the generalized gamma distribution model.

\section{CONCLUSION}

The goal of this study was to explore a simple approach to fit a Generalized Gamma Distribution (GGD) using simulated data, not to propose a new theoretical estimation of the parameters of the generalized gamma distribution. This study shows the efficiency of using the simulated annealing algorithm based on to estimate the parameters of the generalized Gamma distribution for a randomly generated data sample based on the simulation method explained above. The results presented in the tables and figures demonstrated that the parameters of generalized gamma distribution can be optimally estimated using a simulated annealing algorithm incorporated in the maximum likelihood method and the ability to simplify the use of such a generalized distribution to model important phenomena that other distributions cannot. Implementing the simulated annealing algorithm on other distributions from the same family will be our future direction. The study presented in this paper can also contribute to modelling different real-life data set such as internal rate of return and option pricing in the financial market, wind speed and reliability problems in reliability engineering.

\section{ACKNOWLEDGEMENT}

I would like to thank Jazan University, Saudi Arabia for sponsorship opportunity given to me to pursue $\mathrm{PhD}$ at the Universiti Sains Malaysia. I am thankful to my family for their uncounted support and encouraged during my study period. Also, I would like to thank my supervisor who gave me a lot of support and suggestions, and I would also like to thank all the colleagues who encouraged me and gave me valuable advice during my studies.

\section{REFERENCES}

Abbasi, B., A. H. E. Jahromi, J. Arkat, and M. Hosseinkouchack (2006). Estimating The Parameters of Weibull Distribution Using Simulated Annealing Algorithm. Applied Mathematics and Computation, 183(1); 85-93

Abubakar, H. and S. R. M. Sabri (2021a). Incorporating Simulated Annealing Algorithm in The Weibull Distribution for Valuation of Investment Return of Malaysian Property Development Sector. International Journal for Simulation and Multidisciplinary Design Optimization, 12; 22

Abubakar, H. and S. R. M. Sabri (2021b). A Simulation Study on Modified Weibull Distribution for Modelling of Invest- ment Return. Pertanika Journal of Science and Technology, 29(4)

Agarwal, S. K. and J. A. Al-Saleh (2001). Generalized Gamma Type Distribution and its Hazard Rate Function. Communications in Statistics-Theory and Methods, 30(2); 309-318

Amoroso, L. (1925). Ricerche Intorno Alla Curva Dei Redditi. Annali Di Matematica Pura Ed Applicata, 2(1); 123-159

Balakrishnan, N. and Y. Peng (2006). Generalized Gamma Frailty Model. Statistics in Medicine, 25(16); 2797-2816

Bertsimas, D. and J. Tsitsiklis (1993). Simulated Annealing. Statistical Science, 8(1); 10-15

Chen, L., V. P. Singh, and F. Xiong (2017). An Entropy-Based Generalized Gamma Distribution for Flood Frequency Analysis. Entropy, 19(6); 239

Crama, Y. and M. Schyns (2003). Simulated Annealing for Complex Portfolio Selection Problems. European Journal of Operational Research, 150(3); 546-571

Dey, S., E. Garboczi, and A. M Hassan (2021). Method of Moment Analysis of Carbon Nanotubes Embedded in a Lossy Dielectric Slab Using a Multilayer Dyadic Green's Function. TechRxiv

Du, K. L. and M. Swamy (2016). Simulated Annealing. In Search and Optimization by Metaheuristics. Springer; 29-36

Eric, U., O. M. O. Olusola, and F. C. Eze (2020). A Study of Properties and Applications of Gamma Distribution. Statistics, 4(2); 52-65

Franses, P. H. (2016). A Note on The Mean Absolute Scaled Error. International Journal of Forecasting, 32(1); 20-22

Franzin, A. and T. Stützle (2019). Revisiting Simulated Annealing: A Component-Based Analysis. Computers and Operations Research, 104; 191-206

Frías-Paredes, L., F. Mallor, M. Gastón-Romeo, and T. León (2018). Dynamic Mean Absolute Error as New Measure for Assessing Forecasting Errors. Energy Conversion and Management, 162; 176-188

Hirose, H. (1995). Maximum Likelihood Parameter Estimation in The Three-Parameter Gamma Distribution. Computational Statistics and Data Analysis, 20(4); 343-354

Johnson, N. L., S. Kotz, and N. Balakrishnan (1995). Continuous Univariate Distributions Volume 2. John wiley \& sons

Khodabina, M. and A. Ahmadabadib (2010). Some Properties of Generalized Gamma Distribution. Mathematical Sciences

Lawless, J. (2003). Statistical Models and Methods for Lifetime Data. Hoboken, New Jersey: A John Wiley and Sons. Inc

Mead, M., M. M. Nassar, and S. Dey (2018). A Generalization of Generalized Gamma Distributions. Pakistan Journal of Statistics and Operation Research; 121-138

Mudholkar, G. S. and D. K. Srivastava (1993). Exponentiated Weibull Family for Analyzing Bathtub Failure-Rate Data. Institute of Electrical and Electronics Engineers Transactions on Reliability, 42(2); 299-302

Nadarajah, S. and A. K. Gupta (2007). The Exponentiated Gamma Distribution with Application to Drought Data. Calcutta Statistical Association Bulletin, 59(1-2); 29-54

Nagatsuka, H. and N. Balakrishnan (2012). Parameter and 
Quantile Estimation for The Three-Parameter Gamma Distribution Based on Statistics Invariant to Unknown Location. Journal of Statistical Planning and Inference, 142(7); 20872102

Ortega, E. M., G. M. Cordeiro, M. A. Pascoa, and E. V. Couto (2012). The Log-Exponentiated Generalized Gamma Regression Model for Censored Data. Journal of Statistical Computation and Simulation, 82(8); 1169-1189

Orús, R., S. Mugel, and E. Lizaso (2019). Forecasting Financial Crashes with Quantum Computing. Physical Review A, 99(6); 060301

Peng, L. and Q. Yao (2003). Least Absolute Deviations Estimation for ARCH and GARCH Models. Biometrika, 90(4); 967-975

Pradhan, B. and D. Kundu (2011). Bayes Estimation and Prediction of The Two-Parameter Gamma Distribution. Journal of Statistical Computation and Simulation, 81(9); 1187-1198

Sabri, S. R. M. and W. M. Sarsour (2019). Modelling on Stock Investment Valuation for Long-Term Strategy. Journal of Investment and Management, 8(3); 60-66
Sayed, A. and S. Sabri (2022). Transformed Modified Internal Rate of Return on Gamma Distribution for Long Term Stock Investment. Journal of management Information and Decision Sciences, 25(S2); 1-17

Stacy, E. W. (1962). A Generalization of The Gamma Distribution. The Annals of Mathematical Statistics, 33(3); 1187-1192

Tang, L. C. and W. T. Cheong (2004). Cumulative Conformance Count Chart with Sequentially Updated Parameters. Institute of Industrial Engineers Transactions, 36(9); 841-853

Yonar, A. Ş. and N. P. Yapici (2020). A Novel Differential Evolution Algorithm Approach for Estimating The Parameters of Gamma Distribution: An Application to The Failure Stresses of Single Carbon Fibres. Hacettepe Journal of Mathematics and Statistics; 1-22

Zhao, W., J. Li, X. Yang, Q. Peng, and J. Wang (2018). Innovative CFAR Detector with Effective Parameter Estimation Method for Generalised Gamma Distribution and Iterative Sliding Window Strategy. IET Image Processing, 12(1); 6069 